Math. Model. Nat. Phenom.

Vol. 2, No. 2, 2007, pp. 13-19

\title{
Flame Propagation through Large-Scale Vortical Flows: Effect of Equivalence Ratio
}

\author{
L. Kagan and G. Sivashinsky ${ }^{1}$ \\ Sackler Faculty of Exact Sciences, School of Mathematical Sciences, \\ Tel Aviv University, Tel Aviv 69978, Israel
}

\begin{abstract}
The present work is a continuation of previous studies of premixed gas flames spreading through a space-periodic array of large-scale vorticities, and is motivated by the experimentally known phenomenon of flame extinction by turbulence. The prior work dealt with the strongly non-stoichiometric limit where the reaction rate is controlled by a single (deficient) reactant. In the present study the discussion is extended over a physically more realistic formulation based on a bimolecular reaction involving two reactants with different molecular diffusivities, and where the mixture equivalence ratio is utilized as a control parameter. The flow-field is considered as prescribed and unaffected by combustion. The results obtained show that the dual influence of the large-scale turbulence on premixed combustion (flame speed enhancement followed by its reduction and extinction), and higher resilience of turbulent flames at lower Lewis numbers, are not actually related to the multiple-scale nature of the flow-field; the effects may well be captured within the framework of a one-scale flame-flow interaction scheme.
\end{abstract}

Key words:flame-flow interaction, turbulent flames, flammability limits AMS subject classification: $35 \mathrm{~K} 57,80 \mathrm{~A} 32$

\section{Introduction}

Large-scale turbulence is known to exert a dual influence on premixed combustion. Moderately strong turbulence results in the extension of the flame interface and thereby in the burning rate enhancement. Yet it has long been observed that for each gaseous premixture

\footnotetext{
${ }^{1}$ Corresponding author. Email: kaganleo@post.tau.ac.il
} 
there is a certain level of turbulence at which the flame speed reaches its maximum. A further increase in the flow intensity leads to a drop of the flame speed, followed by flame disintegration and extinction [1-3]. Although significant insights have been obtained through many excellent experimental as well as theoretical studies, a first-principle understanding of the phenomenon still remains one of the major challenges of gaseous combustion. The main difficulty in modelling turbulent combustion, as in many others turbulence-related problems, is the wide range of spatio-temporal scales involved. It seems intuitively plausible however, that the multiple-scale nature of the flow-field is not crucial for the physics of flame extinction which may well be described within the framework of a one-scale flame-flow interaction scheme. As has been shown in our previous studies of the problem, one may gain a good deal of apparently relevant information even when the underlying flow is a time-independent and space-periodic array of large-scale vortices [4-6]. The previous work dealt with a strongly non-stoichiometric limit where the reaction rate is controlled by a single (deficient) reactant. In the present study the discussion is extended over a physically more realistic formulation based on a bimolecular reaction involving two reactants with different molecular diffusivities, and where the mixture equivalence ratio is utilized as a control parameter. Other conditions being identical, rich hydrocarbon-air flames are known to be more difficult to quench that lean flames $[2,3]$. It is therefore of interest to ascertain whether this effect has a counterpart in the formulation based on the flame interaction with a space-periodic system of eddies. The adopted reaction-diffusion-advection model involves two diffusion equations for the reactants coupled with the heat equation accounting for radiative heat losses. The flow-field is considered as prescribed and unaffected by combustion. Similarly to the one-reactant model [4-6], it is found that the flame speed is a non-monotonic function of the stirring intensity. For moderately strong vortices, their intensification results in the flame-speed enhancement accompanied by shedding of islands of unburned gas. There is however a certain level of stirring at which the flame speed reaches its maximum. Any further increase in the stirring intensity leads to a drop in the flame speed, followed, for mildly nonadiabatic systems, by flame extinction. In line with experimentally observed tendencies $[2,3]$ it is found that for the same level of stirring, the flame speed enhancement is stronger for the mixtures whose deficient reactant has a higher molecular diffusivity (e.g. rich propane-air or lean hydrogenair mixtures), and such flames appear to be more resilient both against the stirring and heat losses.

\section{Model}

A conventional bimolecular one-step, constant-density, nonadiabatic, reaction-diffusion-advection model for a time-independent vortical flow-field is adopted. In appropriately chosen units the corresponding set of equations for the temperature, fuel and oxidizer concentrations reads,

$$
\begin{gathered}
T_{t}+\mathbf{u} \cdot \Delta T=\Delta^{2} T+(1-\sigma) \Omega-Q \\
\left(C_{F}\right)_{t}+\mathbf{u} \cdot \Delta C_{F}=L e_{F}^{-1} \Delta^{2} C_{F}-\Omega
\end{gathered}
$$




$$
\begin{gathered}
\left(C_{O}\right)_{t}+\mathbf{u} \cdot \Delta C_{O}=L e_{O}^{-1} \Delta^{2} C_{O}-\Omega \\
\Omega=\frac{(1-\sigma)^{3} N^{3}}{4 L e_{F} L e_{O}} C_{F} C_{O} \exp \left(1-\frac{1}{T}\right) \\
Q=h\left(T^{4}-\sigma^{4}\right) \\
\mathbf{u}=(2 A \sin k x \cos k y,-2 A \cos k x \sin k y)
\end{gathered}
$$

Here $T$ is the nondimensional temperature in units of $T_{b}$, the adiabatic temperature of combustion products; $C_{F}$ and $C_{O}$ are the scaled concentrations of the fuel and the oxidizer. In the fresh premixture: $C_{F}=C_{F 0}=2 \varphi /(1+\varphi), C_{O}=C_{O 0}=2 /(1+\varphi)$; where $\varphi$ is the equivalence ratio; is the case of stoichiometry; $x, y$ are the nondimensional spatial coordinates in units of $l_{t h}=D_{t h} / U_{b}$, the thermal width of the flame at $\varphi=1$ and $N>>1 ; D_{t h}$ is the thermal diffusivity of the mixture; $U_{b}$ is the burning velocity of a planar adiabatic flame; $t$ is the nondimensional time in units of $l_{t h} / U_{b} ; \sigma=T_{0} / T_{b}, T_{b}$ is the fresh mixture temperature; $N=T_{a} / T_{b}$ is the nondimensional activation energy; $T_{a}$ is the activation temperature; $L e_{F}=$ $D_{t h} / D_{F}=1.25$ and $L e_{O}=D_{t h} / D_{O}=0.75$ are the Lewis numbers of the fuel and the oxidizer, $D_{F}$ and $D_{O}$ are the molecular diffusivities of the fuel and the oxidizer; $\mathbf{u}$ is the prescribed flow-field and $A=\sqrt{1 / 2<\mathbf{u} \cdot \mathbf{u}>}$ its intensity in units of $U_{b} ; k$ is the periodic flow wave-number in units of $l_{t h}^{-1} ; Q(T)$ is the term responsible for the radiative heat loss, with $h$ being the scaled Stefan-Boltzmann constant referred to $\rho_{b} c_{b} l_{p} U_{b} / 4 T_{b}^{3} l_{t h}$, where $l_{p}$ is the Planck mean absorption length, $c_{p}$ is the specific heat, and $\rho_{b}$ is the burned gas density; $\Omega\left(C_{F}, C_{O}, T\right)$ is the appropriately normalized reaction rate to ensure that at large $N$ and $\varphi=1$ the nondimensional speed of the well-settled planar adiabatic flame is close to unity. The streamlines of the adopted flow-field (6) are depicted on Figure 1.

Equations (2.1)-(2.3) are considered in the strip, $0<x<\pi / k$ and $-\infty<y<\infty$ subjected to the insulating boundary conditions,

$$
T_{x}=\left(C_{F}\right)_{x}=\left(C_{O}\right)_{x}=0 \quad \text { at } x=0, \pi / k
$$

The flame is assumed to propagate upward. Hence the boundary conditions at $y= \pm \infty$,

$$
\begin{array}{cr}
T=\sigma, C_{F}=C_{F 0}, C_{O}=C_{O 0} & \text { at } y \rightarrow+\infty \\
T_{y}=\left(C_{F}\right)_{y}=\left(C_{O}\right)_{y}=0 & \text { at } y \rightarrow-\infty
\end{array}
$$

The problem (2.1)-(2.9) is solved for $N=20, \sigma=0.2, k=0.125$. The computational strategy follows that described in [1].

\section{Numerical Simulations}

\subsection{Adiabatic case}

The normalizing factor $(1-\sigma)^{3} N^{3} / 4 L e_{F} L e_{O}$ in the expression (2.4) for the reaction rate is introduced to ensure that in the limit of infinitely high activation energy $(N \rightarrow \infty)$ the scaled 


\begin{tabular}{|c|c|c|c|c|}
\hline$\varphi$ & 0.8 & 0.9 & 1.0 & 1.1 \\
\hline Mixture & $44 \% \mathrm{~F}+56 \% \mathrm{O}$ & $47 \% \mathrm{~F}+53 \% \mathrm{O}$ & $50 \% \mathrm{~F}+50 \% \mathrm{O}$ & $52 \% \mathrm{~F}+48 \% \mathrm{O}$ \\
\hline$V_{0}$ & 0.582 & 0.808 & 0.872 & 0.738 \\
\hline
\end{tabular}

Table 1: Mixture composition and speed of a planar adiabatic flame.

propagation speed of a planar adiabatic stoichiometric $(\varphi=1)$ flame is held at unity. For the high but finite activation energy $(N=20)$ employed in the numerical simulations there is naturally a deviation of $V_{0}$ from unity. Some representative values of $V_{0}(\varphi)$ at $N=20$, $\sigma=0$ and $h=0$ are given in Table 1 . These numbers are obtained by numerical simulations of the one-dimensional version of the model (2.1) - (2.9) at $\mathbf{u}=0$.

Figure 1 shows the distributions of temperature $(T)$, concentrations of fuel $\left(C_{F}\right)$ and oxidizer $\left(C_{O}\right)$ at $\varphi=1.1$ and $A=7.5$, where the flame speed reaches its maximum (Figure 2). The distributions are similar to those found for the single reactant model [4]. Moreover, the distributions for both reactants look alike despite different diffusivities: for the fuel $L e_{F}=1.25$ and for the oxidizer $L e_{O}=0.75$.

Figure 1 also shows how the flame envelops the island of the unburned gas. These islands eventually burn out far behind the leading edge of the advancing flame. Moreover, upon some transient period (corresponding to the passage through approximately two vortex cells) the distributions of temperature and concentration become nearly constant along the streamlines. A similar effect is discussed in [7]. In order to single out the impact of equivalence

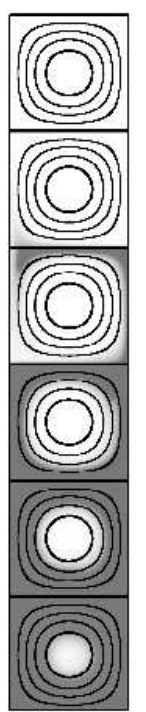

$T$

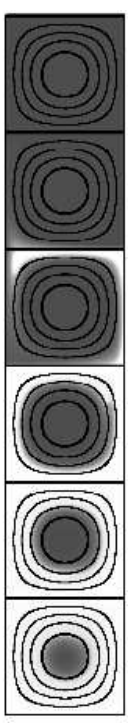

$C_{F}$

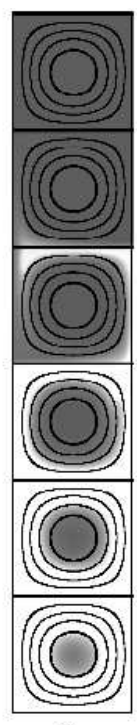

$C_{O}$

Figure 1: Distributions of temperature $(T)$, concentrations of fuel $\left(C_{F}\right)$ and oxidizer $\left(C_{O}\right)$ for $\varphi=1.1$ and $A=7.5$. Darker shading corresponds to higher levels of $T, C_{F}$ and $C_{O}$.

ratio in its 'purest' form, in the further discussion we deal with the renormalized flame speed $\bar{V}$ referred to $V_{0}(\varphi)$ (Table 1.). Figure 2 depicts $\bar{V}$ versus $A$ curves calculated for several 


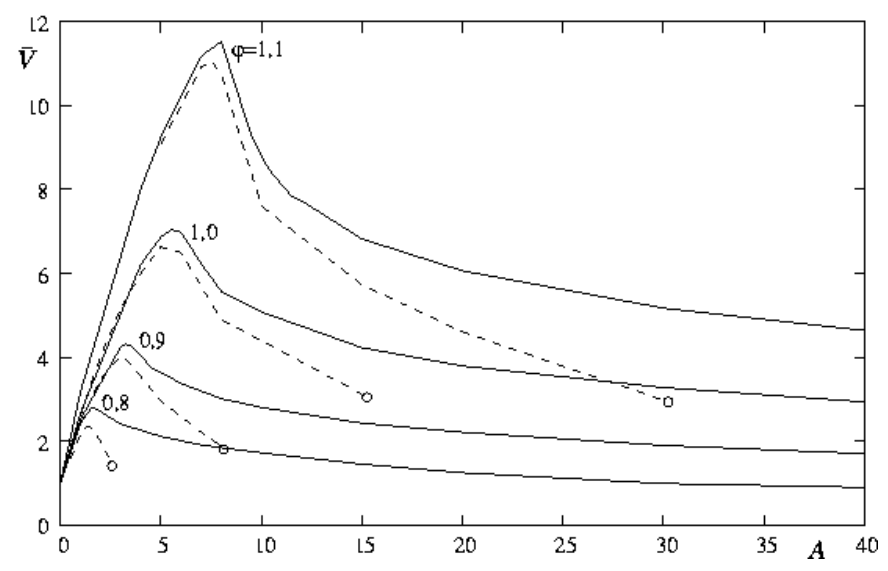

Figure 2: Normalized flame speed $(\bar{V})$ versus flow-intensity $(A)$. Solid and dashed lines correspond to the adiabatic and non-adiabatic cases, respectively. In the non-adiabatic case $h=0.002$. Open circles mark the extinction points.

equivalence ratios at $h=0$ (solid lines) and $h=0.002$ (dashed lines). As expected [4-6], the emerging dependencies are nonmonotonic. At relatively weak stirring its intensification results in the flame speed enhancement. Yet, there is a crossover intensity $A_{c r}(\varphi)$ where the flame speed reaches its maximum. As is readily seen $A_{c r}(\varphi)$ is an increasing function of the equivalence ratio $\varphi$, which agrees with the experimentally observed tendencies [1-3]. At $A<<A_{c r}$ the curves $\bar{V}(A)$ nearly coincide. This conforms with the Huygens model [4], valid in the limit of large-scale vortices $(k<<1)$, and where the flame structure is locally planar and unaffected by the underlying flow-field. Figure 2 also shows that for the same stirring intensity the flame propagates faster if the Lewis number of the deficient reactant is smaller. For $\varphi=1.1$ the deficient reactant is the oxidizer $\left(L e_{O}=0.75\right)$, whereas for $\varphi=0.9$ the deficient reactant is the fuel $\left(L e_{F}=1.25\right)$. The flame propagates significantly faster for $\varphi=1.1$ than for $\varphi=0.9$ (Figure 2), which is perfectly in line with [6].

\subsection{Nonadiabatic Case.}

For all the intricacies of the flame-vortical flow interaction, the adiabatic flame seems to withstand any level of stirring however high. Incorporation of volumetric heat losses changes the picture essentially. Figure 2 also shows the $\bar{V}(A)$ dependencies in the presence of heat losses $(h=0.002)$. This value of $h$ ensures the existence of the planar flame spreading through the quiescent premixture $(A=0)$ at all equivalence ratios considered. As expected [4-6], heat losses exert an inhibiting influence on combustion resulting in reduction of the flame speed, and complete extinction at sufficiently strong stirring. For $A<<A_{c r}$ the impact of the heat losses on the flame speed is almost negligible. The Huygens model therefore is valid also for mildly non-adiabatic flames, at least far from the extinction point (Fig. 2). The maximum stirring intensity (just below the extinction point) grows with the equivalence ratio. This effect was also observed experimentally $[2,3]$. It is clear that for each equivalence 


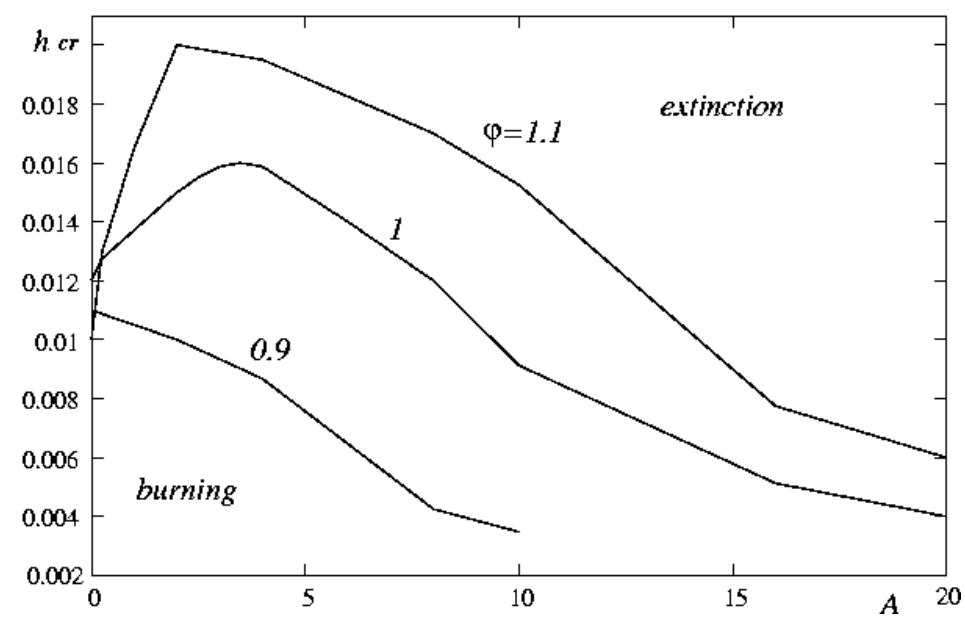

Figure 3: Threshold heat loss $\left(h_{c r}\right)$ versus vortex intensity A evaluated for several equivalence ratios.

ratio and flow intensity a strong enough heat loss extinguishes the flame, i.e. there is certain critical level of the heat loss $\left(h_{c r}\right)$ above which the flame spread is ruled out. Figure 3 plots $h_{c r}(A)$ dependencies evaluated for several equivalence ratios. Since $V_{0}$ reaches maximum at $\varphi=1$ (Table 1 ) the planar flame $(A=0)$ of the stoichiometric mixture $(\varphi=1)$ is more resilient against heat losses. To suppress faster flames one needs a higher level of heat losses. Nonmonotonicity of $h_{c r}$ is apparently a reflection of that of $\bar{V}(A)$.

\section{Concluding Remarks}

- The nonmonotinicity of the $\bar{V}(A)$ dependency (Fig. 2) does not exhaust all the phenomenology contained in the model. As evidenced by single reactant models [5], moving to higher levels of stirring the adiabatic flame may begin to speed up again. Although this (third) propagation mode is likely to be out of reach for practical gaseous flames because of quenching considerations, it is likely to be of relevance for isothermal autocatalytic reaction waves characterized by a marked persistence of the $\bar{V}(A)$ monotonicity [8]. As discussed in Ref. [5], the basic reason for the marked distinction between the two systems is the strong sensitivity of the reaction rate to variation in temperature (i.e. high activation energy) in gaseous flames and its relatively mild response to change in the concentration of autocatalytic products in isothermal waves.

- The two-reactant model employed reproduces the experimental observation that rich hydrocarbon-air mixtures are more difficult to quench than lean ones, with an opposite to effect for hydrogen-air mixtures [2,3].

- In all cases considered the spatial interval $(0<x<\pi / k)(2.7)$ is not wide enough for the flame front to display the diffusive-thermal instability which, since $L e_{O}<L e_{F}$, 
occurs in fuel-rich mixtures $(\varphi>1)$. The instability may somewhat alter the character of the $\bar{V}(A)$ and $h_{c r}(\mathrm{~A})$ dependencies (see also [6]). More research on this question is needed.

- As a natural next step in simulating turbulent flames it would be interesting to extend the present analysis over multiple-scale flow-fields.

- In the present constant-density model, the effect of the flow-field distortion by the temperature field created by the flame is ignored. It would be important to ascertain how the found $\bar{V}$ vs. $A$ response is altered when there is a coupling between the flame and the flow-field.

\section{Acknowledgements}

The authors gratefully acknowledge the support of the U.S.-Israel Binational Science Foundation (Grant 2006-151), the Israel Science Foundation (Grants 350/05), and the European Community Program RTN-NPRN-CT-2002-00274.

\section{References}

[1] D. Bradley. How fast can we burn? Proc. Combust. Inst., 1999, Vol. 24, 247-262.

[2] V.P. Karpov, E.S. Severin. Effects of molecular-transport coefficients on the rate of turbulent combustion. Combust. Expl. Shock Waves, 1980, Vol 16, 41-46.

[3] R. G. Abdel-Gayed, D. Bradley, M. N. Hamid, M. Lawes. Lewis number effects on turbulent burning velocity. Proc. Combust. Inst., 1984, Vol. 20, 505-512.

[4] L. Kagan, G. Sivashinsky. Flame propagation and extinction in large-scale vortical flows. Combust. Flame, 2000, Vol. 120, 222-232.

[5] L. Kagan, P.D. Ronney, G. Sivashinsky. Activation energy effect on flame propagation in large-scale vortical flows. Combust. Theory Modelling, 2002, Vol. 6, 479-488.

[6] L. Kagan, G. Sivashinsky. Effect of Lewis number on flame propagation through vortical flows. Combust. Flame, 2005, Vol. 142, 235-240.

[7] L. Kagan, H. Berestycki, G. Joulin, G. Sivashinsky. The effect of stirring on the limits of thermal explosion. Combust. Theory Modelling, 1997, Vol. 1, 97-112.

[8] N. Vladimirova, P. Constantin, A. Kiselev, O. Ruchanskiy, L. Ryzhik. Flame enhancement and quenching in fluid flows. Combust. Theory Modelling, 2003, Vol. 7, 487-508. 